\title{
Djinn als Instrumente der Sicherheit
}

\author{
Wertorientierung in einer komplexen Welt durch Unwesen und Schabernack der Dämonen
}

Sie bilden so etwas wie eine Parallelwelt. Einerseits eine Spiegelung, aber auch eine Ergänzung zu den im allgemeinen sichtbaren Phänomenen. Manchmal treten sie freilich selbst in Erscheinung und ganz bestimmt sind ihre Spuren in der sogenannten Wirklichkeit bemerkbar: Die Djinn. Dämonen, die in vielen Kulturen ihr Unwesen und ihren Schabernack treiben - und bis heute eine Form von Bewältigungsstrategien unangenehmer und nicht immer logisch nachvollziehbarer Ereignisse darstel-

len. Für die Region des ländlichen Syrien hat der an der Österreichischen Akademie der Wissenschaften tätige Sozialanthropologe Dr. Gebhard Fartacek in jahrelangen umfassenden Feldstudien diese Gegenwelt untersucht, hat mit den Menschen lange Gespräche über ihre Begegnungen mit den eigenwilligen, übernatürlichen Geschöpfen geführt und diese analysiert. Kürzlich erschien der aktuelle Stand seiner Forschungen in Buchform.

Die Existenz der Djinn begründet sich zwar auch im Koran, sie bilden aber unabhängig von der Religion einen Lebensbestandteil in vielen arabischen Gesellschaften und sind auch ein faszinierender Gegenstand der Forschung. Die Sagenwelt des großen und vielfältigen Kulturkreises, die eine Erklärungsmöglichkeit für schlechte Erfahrungen, Unglücksfälle, Krankheiten und ähnliches bietet. Ein personalisiertes Muster, um Komplexität zu reduzieren. Dabei können sie sehr unterschiedliche Formen annehmen - als menschliche Gestalt mit ganz bestimmten Merkmalen, als Tier oder auch als Pflanze und sie können indirekt Einfluss ausüben: Über den bösen Blick und die schwarze Magie beispielsweise. Interessanterweise treten Djinn, deren es ganz unterschiedliche Arten gibt, und damit verbundene Erscheinungen häufig an zeitlichen und örtlichen Übergängen auf: Unter Türschwellen oder Brücken, aber auch in speziellen Situationen wie bei der Geburt eines Kindes oder bei der Hochzeit und in den meisten Fällen geht es letztlich um Wertfragen. Sie entsprechen der Zeit und dem Ort. „Begegnungen mit Djinn, wie sie in der heutigen Arabischen Republik Syrien stattfinden und diskutiert werden, sind auf substanzielle Weise mit den aktuellen Problemen und Bedürfnissen der Menschen verbunden“, stellt Fartacek fest. Sie können als Ergebnis von Ungewissheiten interpretiert werden, die mit ethisch-moralischen Entscheidungen sowie mit der Konstruktion von Gender und Identität einhergehen.

Um also den schlechten Einfluss der Dämonen zu beseitigen oder diesem überhaupt schon vorzubeugen, stehen verschiedene Strategien zur Verfügung. Religiöse Experten können mit bestimmten Ritualen, die „Besessenen“ heilen - beispielsweise mit Koranzitaten, die möglicherweise eine hyp-

\section{Buchempfehlung}

24 Fallbeispiele - Geschichten - verdeutlichen das Wirken der Djinn und wie man sich dagegen zur Wehr setzen kann. Eine kompakte schlüssige Analyse, ebenso wie die theoretische Einordnung öffnen eine faszinierende Welt, in der sich manche Parallelen aber auch viele Anknüpfungspunkte für neue Möglichkeiten finden lassen. Spannend und tatsächlich vergnüglich zu lesende seriöse Forschung. notische oder suggestive Kraft entfalten. Ausräuchern eines von Djinn bewohnten Raums, das Nichtaussprechen bestimmter Begriffe oder das Sprechen ritualisierter Redewendungen oder auch das Tragen von Amuletten kann das Problem ebenso kontrollieren wie die Zubereitung eines guten Essens, um den Djinni zu besänftigen. Die Möglichkeit, handeln zu können, bedeutet für den Menschen im allgemeinen größere Sicherheit.

Die gedankliche und durchaus reale Beschäftigung mit den Djinn führt die Menschen an die konfliktträchtigen Themen Ethik und Moral, Sexualität, sozialer Wandel und Identität, stellt der Sozialanthropologe in seiner Schlussfolge-

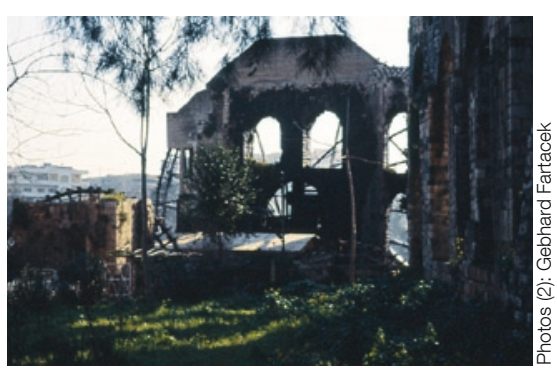

oder in antiken Stätten.

rung fest. Auf diesem Weg ergeben sich wirksame Strategien für ein richtiges und gutes Leben, die im Zusammenhang der lokalen Gegebenheiten ihre Gültigkeit haben und natürlich auch Identitätsstiftend sind. Konfrontiert mit überregionalen Einflüssen sieht Fartacek in diesen lokalkulturellen Konzeptionen aber ein dynamisches und zukunftsorientiertes Instrument der Wertklärung, das bei den heiklen und wichtigen Themen zum Einsatz kommt. Im Gegensatz zum westlichen Denken lassen diese Instrumente eine Vielzahl an - manchmal durchaus widersprüchlich scheinenden Möglichkeiten zu und suchen letztlich Konsens und Ausgleich.

Gebhard Fartacek: Unheil durch Dämonen? Geschichten und Diskurse über das Wirken der Ğinn. Eine sozialanthropolgische Spurensuche in Syrien, Böhlau 2010, 216 Seiten mit 32 farbigen Abbildungen, ISBN-13: 9783205784852 , Preis: 39,00 Euro 